\title{
Management of Hypocalcemia After Modified Bilateral Radical Neck Dissection Followed with Total Thyroidectomy: A Case Report
}

\author{
I Made Arimbawa ${ }^{1}$, Ayu Diah Perdana Paramita² ${ }^{2}$ I Gde Ardika Nuaba ${ }^{3}$, Herman Saputra $^{4}$ \\ Manuscript submitted: 27 November 2020, Manuscript revised: 09 December 2020, Accepted for publication: 18 January 2021
}

*Corresponding Author

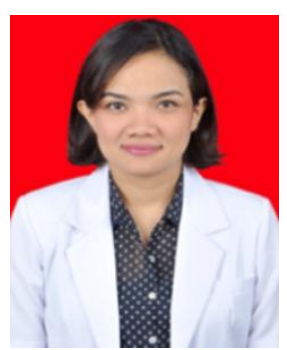

\section{Keywords}

calcitriol;

calcium;

hypocalcemia;

hypoparathyroidism;

hypovitaminosis;

\begin{abstract}
Hypocalcemia is a common complication after total thyroidectomy due to acute hypoparathyroidism post-surgery. The incident varies from 7\%-69\% depends on the surgery center. The management is divided based on the onset and symptoms. Carpal-pedal spasm, seizure, bronchospasms are the emergency symptoms of hypocalcemia that require administration of intravenous calcium. However, the management of asymptomatic hypocalcemia with oral calcium, vitamin $\mathrm{D}$, and hydrochlorothiazide can be difficult due to wide fluctuations of serum calcium and the risks of complication. Hereby we report a 14-year-old girl with hypocalcemia due to hypoparathyroidism post modified bilateral radical neck dissection and total thyroidectomy. She had papillary thyroid carcinoma and lymph node metastases. After surgery, she developed classic signs of hypocalcemia, numbness in extremities which progressed to cramps. Physical examination revealed chvostek's sign. Laboratory examination showed the critical value of hypocalcemia with a normal level of albumin, hypoparathyroidism, and hypovitaminosis $\mathrm{D}$. The initial treatment was started by giving intravenous calcium gluconate $10 \%$ and calcitriol. Monitoring levels of calcium and phosphate were performed regularly. Calcium level was maintained by giving a high dose of elemental calcium, vitamin D3 and hydrochlorothiazide followed.
\end{abstract}

International Journal of Health Sciences (C) 2021. This is an open access article under the CC BY-NC-ND license (https://creativecommons.org/licenses/by-nc-nd/4.0/).

\footnotetext{
${ }^{1}$ Department of Child Health, Medical Faculty, Udayana University, Indonesia

${ }^{2}$ Department of Child Health, Medical Faculty, Udayana University, Indonesia

${ }^{3}$ Department of Ear, Nose, Throat-Head and Neck Surgery, Medical Faculty, Udayana University, Indonesia

${ }^{4}$ Department of Pathological Anatomy, Medical Faculty, Udayana University, Indonesia
} 


\begin{tabular}{|c|c|c|}
\hline \multicolumn{3}{|c|}{ Contents } \\
\hline \multicolumn{3}{|c|}{ Abstract........ } \\
\hline & Introduction... & 10 \\
\hline & Case Report ..... & 11 \\
\hline 3 & Discussion ............................................... & 14 \\
\hline & Conclusion & 17 \\
\hline & (1, & 17 \\
\hline & 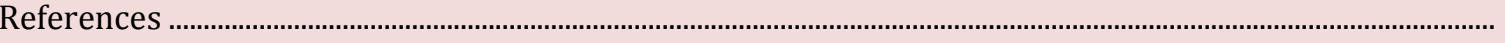 & 18 \\
\hline & Biography of Authors ............................... & 19 \\
\hline
\end{tabular}

\section{Introduction}

Hypocalcaemia is a common complication of central neck surgery especially in thyroid surgery (Mejia et al., 2018; Dedivitis et al., 2017; Boumgarten et al., 2019). It is due to the trauma of parathyroid glands caused by direct injury during surgery, devascularization caused by obstruction of venous drainage, or incidental parathyroidectomy (Dedivitis et al., 2017). This trauma will cause hypoparathyroidism (HPT) associated with low or no production of parathyroid hormone and characterized by hypocalcemia, hyperphosphatemia. The hypocalcemia post thyroid surgery incident varies from $7 \%$ to $69 \%$ depends on the pediatric surgery center (Boumgarten et al., 2019). The risk factor for HPT post thyroid surgery including: surgery of the bilateral central neck, surgery of malignancy in the thyroid, surgery of parathyroid hyperplasia, central compartment lymphadenectomy due to metastases, surgeon expertise, surgical technique, duration of the surgery, deficiency or insufficiency of vitamin D pre-surgery, prior gastric bypass surgery, female, pregnancy, and lactation (Boumgarten et al., 2019; Stack et al., 2015).

Hypoparathyroidism after thyroid surgery can be classified into transient HPT or permanent HPT, based on the resolution time of hypocalcemia. The hypocalcemia in transient HPT would improve between 6 to 12 months after surgery, meanwhile, the hypocalcemia in permanent HPT would last longer than 12 months after neck surgery (Mejia et al., 2018; Di Maio et al., 2018). The prevalence of transient HPT is $27 \%$ and the permanent HPT is $1 \%$ (Dedivitis et al., 2017).

Biochemically, the hypoparathyroidism after thyroid surgery is defined as hypocalcemia, the CSC value $<8$ $\mathrm{mg} / \mathrm{dL}$ (with or without hypocalcaemia symptoms) and PTH $<15 \mathrm{pg} / \mathrm{mL}$. The ideal protocol to assess HPT after surgery is measure the level of calcium, albumin and PTH in the first 24 hours after surgery. Patient can be classified as 1) low risk (CSC 8-8.5 mg/dL, PTH > $15 \mathrm{pg} / \mathrm{mL}$ ); 2) medium risk (CSC 7.5-8 mg/dL or PTH 6$15 \mathrm{pg} / \mathrm{mL}$ ); 3) high risk ( $\mathrm{CSC}<7.5 \mathrm{mg} / \mathrm{dL}$ and PTH $<5 \mathrm{pg} / \mathrm{mL}$ ). This classification is useful for planning supplementation of calcium, vitamin D, thiazide diuretic, discharge and laboratory evaluation (Mejia et al., 2018; Di Maio et al., 2018).

Symptoms of hypocalcemia appear like the effect of low calcium serum in the organ and the acuity of the onset. In acute onset, the neurological symptoms can be described as paresthesia in the perioral region, hands and feet progressing to carpopedal spasm, defined as an involuntary muscle contraction with pain, hyperreflexia, and muscle spasm. The chvostek's sign on the face (present in 94\% of cases of true acute hypocalcemia) and trousseau's sign, both are the classical sign in the neurological examination. In severe cases of hypocalcemia, death can occur due to laryngospasm, bronchospasm, seizure, change of cardiac contractility, and cardiac electrical conduction (Mejia et al., 2018; Stack et al., 2015).

The management of hypocalcemia in HPT is divided based on its symptoms and onset. The symptomatic acute hypocalcemia like a carpopedal spasm, seizure, bronchospasm, laryngospasm, and angina pectoris need intravenous administration of calcium and the chronic management of HPT by using oral calcium and vitamin D supplementation, thiazide diuretics to maintain the asymptomatic hypocalcemia and avoid the complication of overtreatment which may lead to nephrolithiasis, nephrocalcinosis and renal insufficiency (Mejia et al., 2018; Stack et al., 2015; Di Maio et al., 2018). We present a case of hypocalcemia which presented in a girl who previously had a total thyroidectomy. The objective of this case report is to describe the management of hypocalcemia in patients who have undergone total thyroidectomy (Abboud et al., 2002; Reinhardt et al., 2011). 


\section{Case Report}

A fourteen years old girl had a mass in the anterior neck that was realized a year ago. The mass was solid with large nodules that were noticed as a palpable mass. The patient did not realize when the mass showed up for the first time because it was painless however it was getting bigger. The patient did not have either difficulty in swallowing, pressure on her neck, or shortness of breath while lying. The patient also denied had nervousness, anxiety, heat intolerance, hyperactivity, palpitation, tremor, weight loss, muscle weakness and can perform the regular activity. The patient had several clinical examinations since August 2018 and was diagnosed with papillary thyroid carcinoma extra. The patient was planned to have a total thyroidectomy on November $25^{\text {th }} 2019$.

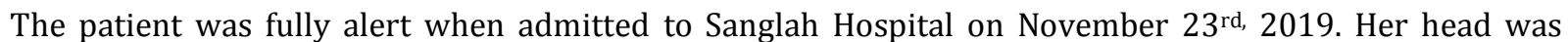
normal in shape. There was no sign of hyperthyroidism like exophthalmos, lid lag, lid retraction, conjunctiva injection, chemosis, nor periorbital edema. The conjunctiva was not pale and the light reflex pupil was normal and the size was equal. The ears, nose, and throat examination were within normal limits. There was a solid mass on the anterior neck which was not visible (figure 1) with multiple nodules in palpation especially on her right neck. There was multiple lymph nodes enlargement on her right neck and one lymph node enlargement on her left neck. Her chest was symmetrical both on rest and movement. The breath sound was vesicular without rales or wheezing. The first and second heart sounds were normal, regular without murmur in auscultation. There was no lymph node enlargement on both axillae. The abdomen was not distended. Bowel sound was normal when auscultated. The liver and spleen were no palpable. Skin turgor was normal without smooth sweaty skin. There was no edema on extremities or lymph nodes enlargement on both inguinal. The anal and genital examination was normal.

The hemodynamics of this patient were within normal limit with blood pressure $100 / 70 \mathrm{mmHg}\left(\mathrm{P}_{50}\right)$, heart rate 78 beats per minute, skin temperature of $36,8^{\circ} \mathrm{C}$. Patient's body weight was $49 \mathrm{~kg}$ and body height was $153 \mathrm{~cm}$, with father's height was $168 \mathrm{~cm}$ and mother's height was $160 \mathrm{~cm}$. Her arm span was $158 \mathrm{~cm}$ and the upper body segment was $76 \mathrm{~cm}$. The growth factor of the patient according to her height for age (HFA, CDC 2000) was within 10-25 percentile and her height was still within mid parental height with the upperlower segment was 0,98 . There was no sign of short stature or accelerated growth according to her anthropometry.

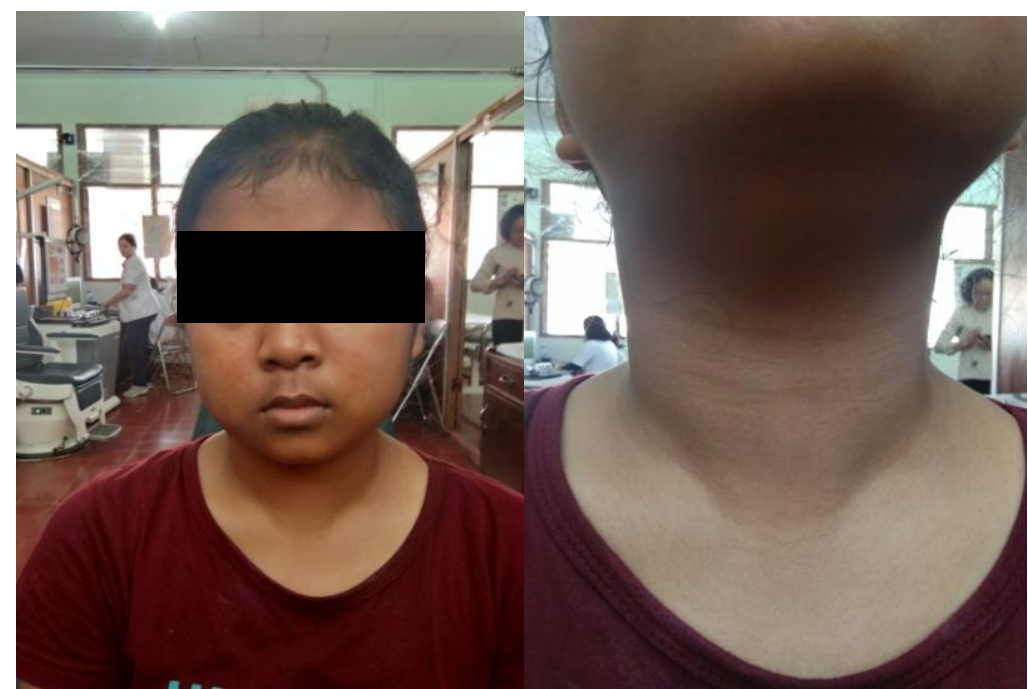

Figure 1. The mass on the anterior neck that was not visible

Arimbawa,. I. M., Paramita, A. D. P., Nuaba, I. G. A., \& Saputra, H. (2021). Management of hypocalcemia after modified bilateral radical neck dissection followed with total thyroidectomy: a case report. International Journal of Health Sciences, 5(1), 9-19. https://doi.org/10.29332/ijhs.v5n1.654 
Laboratory test before surgery revealed a normal limit in blood purifier analysis, albumin was 4,6 g/dL, normal function of renal and liver, and normal level of potassium and sodium. There were no calcium, PTH, and vitamin D serum evaluation before the operation. Thyroid ultrasound revealed multiple nodules on the right thyroid with hypervascularization, which was suspected of malignancy. Contrast study of neck region showed multiple hypodense nodules on right thyroid lobes and isthmus with calcification in the right thyroid lobes and enhancement in contrast; multiple lymphadenopathies in heterogenic density on submandibular, coli and supraclavicular that lobulated, firm in shape, regular, with the biggest size $+3,0 \times 4,5 \times 7,5 \mathrm{~cm}$ in right coli and enhancement in contras (figure 2). Histopathology examination from fine-needle aspiration biopsy (FNAB) suggested metastases of papillary thyroid carcinoma. The patient is the second child in the family who lives in the urban area in Denpasar, with no history of thyroid or other types of cancer in her family and no history of radiation exposure.

During the surgical procedure, the ENT surgeon found multiple lymph nodes enlargements bilateral in levels 1, 2, 3, 4, and 6 and solid mass in thyroid dextra and Sinistra (figure 3). The modified radical neck dissection bilateral was done followed by total thyroidectomy. The parathyroid gland was identified and was not taken during the operation. There was no bleeding occurred during and post-operation. It took a total of 5 hours and 45 minutes operating time. A histopathologic examination from the mass was consistent with papillary thyroid carcinoma tall cell variant (figure 4 ).

The patient was admitted to Pediatric Intensive Care Unit (PICU) after surgery for evaluation. She was given ceftriaxone intravenously as a prophylaxis antibiotic, sodium thyroxin 0,1 mcg every 24 hours, methylprednisolone $62,5 \mathrm{mg}$ every 12 hours, codeine, and $\mathrm{N}$-acetylcysteine as an analgesic and calcium carbonate $500 \mathrm{mg}$ every 24 hours for prophylaxis of hypocalcemia post neck surgery. The patient moved to the pediatrics general ward after 24 hours in PICU. Thirty-six hours after surgery, the patient developed numbness, localized on her hands and feet, progressed into cramps with pain which is known as carpopedal spasm, and chvostek's sign was positive, which was noticed as momentarily contraction when there was a trigger at the angle of the jaw. The patient did not have a seizure or psychological and behavioral changes, she also did not complain of chest pain. The patient did not have difficulty breathing when the symptoms appeared patient developed hypotension with blood pressure 90/60 $\mathrm{mmHg}\left(<\mathrm{P}_{50}\right)$.

Laboratory test after the symptoms occurred, revealed the critical value of hypocalcemia $5.7 \mathrm{mg} / \mathrm{dL}$, normal albumin $3.80 \mathrm{~g} / \mathrm{dL}$, hypomagnesium $1.43 \mathrm{mg} / \mathrm{dL}$, hypo vitamin D 25-OH 11.73 ng/mL, and hypoparathiroid hormone $1.37 \mathrm{pg} / \mathrm{mL}$ which occurred 3 days later. The patient was given acute management for hypoparathyroidism due to thyroid surgery. The intravenous calcium gluconate $10 \%$ was given in adult dose, $100 \mathrm{mg} / \mathrm{kg} /$ day every 8 hours followed by continuous intravenous infusion of $1 \mathrm{mg} / \mathrm{kg} / \mathrm{hour}$. The level of calcium was increased to $6.6 \mathrm{mg} / \mathrm{dL}$ on the $3^{\text {rd }}$ day of calcium correction. The patient was given calcium carbonate $3000 \mathrm{mg} /$ day and calcitriol 0,04 $\mu \mathrm{g} / \mathrm{kg} /$ day and continuous intravenous infusion of $1 \mathrm{mg} / \mathrm{kg} / \mathrm{hour}$ until the level of calcium reached $>7,5 \mathrm{mg} / \mathrm{dL}$. The CSC was $7.8 \mathrm{mg} / \mathrm{dL}$ on the $8^{\text {th }}$ day after surgery. Her length of stay was prolonged to 8 days due to repeated hypocalcemia. Finally, the patient was discharged with mild numbness on their hands and feet. After discharge from the hospital, the patient continued the treatment by using oral calcium carbonate 3,000 mg/day, calcitriol $2 \mathrm{mcg} / \mathrm{day}$, hidroclorotiazid $25 \mathrm{mg} / \mathrm{day}$, and was advised to take vitamin D3 2,000 IU/day.

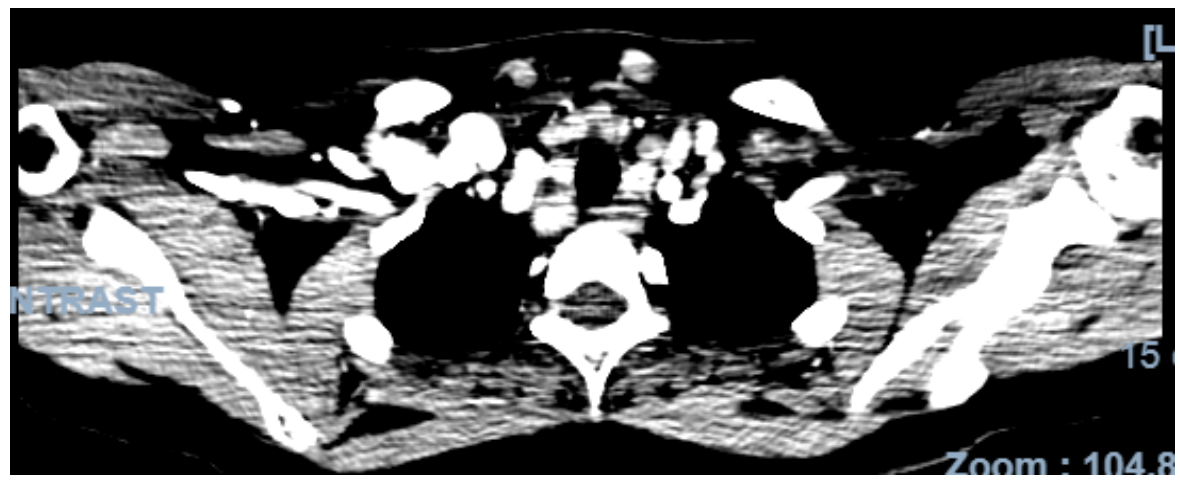




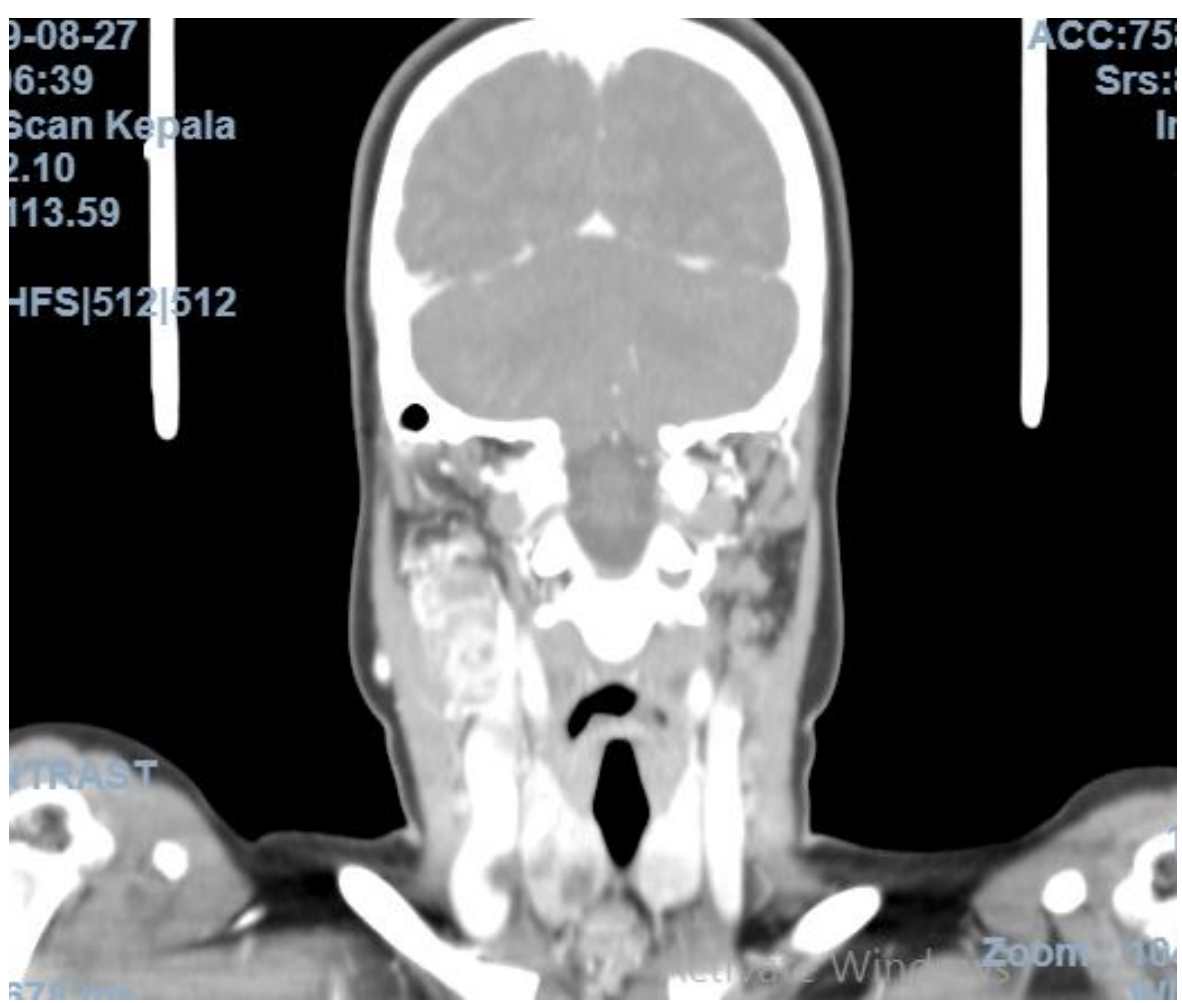

Figure 2. CT-Scan of the neck region showed multiple nodules in right thyroid lobes and isthmus with multiple lymphadenopathies in submandibular, coli, and supraclavicular bilateral

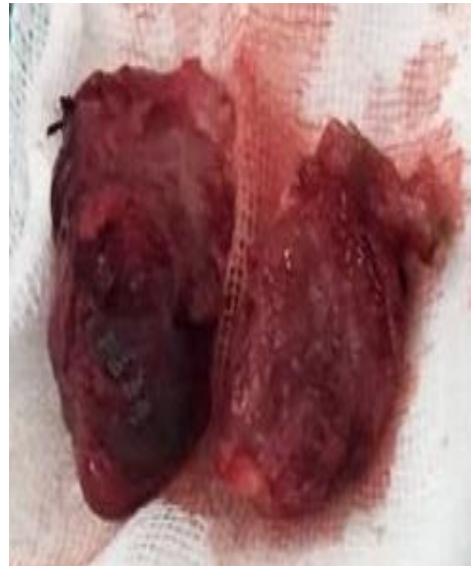

3 (a)

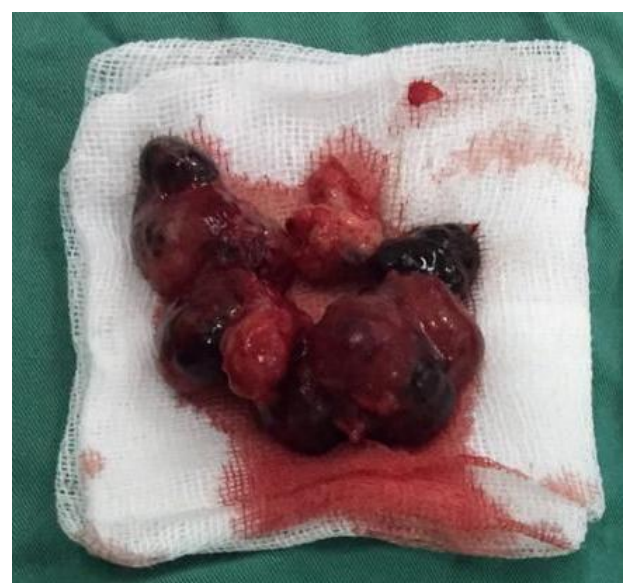

3 (b)

Figure 3. (3a) Solid mass on left and right thyroid; (3b) multiple lymphadenopathies

Arimbawa,. I. M., Paramita, A. D. P., Nuaba, I. G. A., \& Saputra, H. (2021). Management of hypocalcemia after modified bilateral radical neck dissection followed with total thyroidectomy: a case report. International Journal of Health Sciences, 5(1), 9-19. https://doi.org/10.29332/ijhs.v5n1.654 


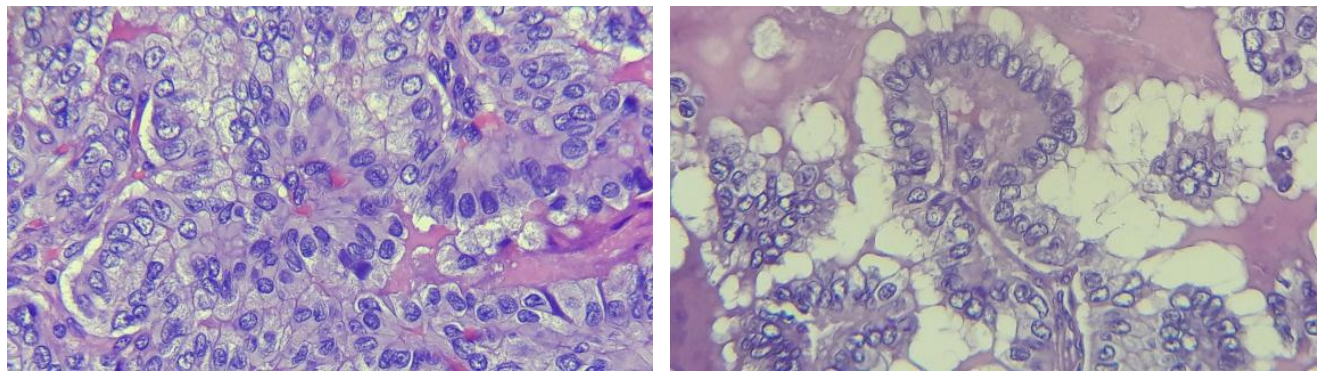

Figure 4. A histopathologic examination from mass showed proliferation and papillary growth pattern of epithelial cell, crowding, overlapping in a bigger size, with large eosinophilia cytoplasmic, ground-glass appearance, and nuclear groove. It is consistent with papillary thyroid carcinoma tall cell variant

One week after discharge, the patient-controlled to the pediatric endocrine outpatient clinic and was given calcium citrate that has a lower level of elemental calcium but has better absorption, high dose of vitamin D3 meanwhile calcitriol was discontinued. One month after surgery, the calcium serum did not reach the target (the lowest normal range) even though the PTH was increased to $3.85 \mathrm{pg} / \mathrm{mL}$. Since hypercalciuria was not found in urine electrolytes examination, the patient was recommended to use elemental calcium from calcium carbonate 3,000 mg/day in 3 divided doses, vitamin D3 4,000 IU/day in 2 divided doses, and hydrochlorothiazide $25 \mathrm{mg}$ daily. Two months after surgery, the vitamin D 25-0H serum was increased from $11.73 \mathrm{ng} / \mathrm{mL}$ to $68.7 \mathrm{ng} / \mathrm{mL}$. Six months after the surgery, the CSC level was $9.2 \mathrm{mg} / \mathrm{dl}$, following target which was a lower level of normal value and the phosphate was $5.39 \mathrm{mg} / \mathrm{dL}$. In the present day, the patient continues the therapy for the preparation of radio ablation with elemental calcium 2,000 $\mathrm{mg} /$ day in 3 divided doses, vitamin D3 2,000 IU/day in 2 divided doses, and hydrochlorothiazide $25 \mathrm{mg}$ daily.

\section{Discussion}

Papillary thyroid carcinoma is the most common type of thyroid cancer, with an estimation of about $80-85 \%$ of all cases of thyroid cancer (Aboelnaga \& Ahmed, 2015). It affects all ages but mostly in the adult patient and the prognostic is better if it is diagnosed before the age of 40 . The first symptom of thyroid carcinoma is thyroid nodule. In the beginning, it is a painless, asymptomatic lump in the neck, usually detected after a routine examination. When it is found in a patient younger than 20 years old, $25 \%$ of thyroid nodule was diagnosed as cancer (Limaiem et al., 2019). The other symptoms like pain in the anterior neck and changes in voice may occur later (Aboelnaga \& Ahmed, 2015). Compressive symptoms including the difficulty of swallowing, pressure, and shortness of breath when lying flat occur later when the nodule became bigger. Big thyroid nodules are concerning for cancer include those that are non-tender, firm, growing, and does not shrink over time.

The risk factors for papillary thyroid cancer include radiation exposure to the neck and head and family history of thyroid cancer but the majority of patients have no risk factors (Maxwell et al., 2017). The best diagnostic procedure for thyroid nodules is fine-needle aspiration biopsy (FNAB), neck CT scan, or neck MRI. The sensitivity of FNAB to diagnose papillary thyroid cancer is almost $80 \%$ and the specificity is almost $100 \%$ (Maxwell et al., 2017). In this case, the patient was a 14 years old girl who realized a lump in her neck after palpated the mass in her neck, there was no complaint about the mass because there was no pain, no difficulty to swallow, no difficulty to breath. There was neither history of radiation exposure when she was a young nor genetic factor in her family. The patient was examined with the ultrasound of the thyroid and CT-scan of the neck region with contrast, the result showed multiple nodules with hypervascularisation in thyroid dextra and multiple lymphadenopathies. The result of FNAB suggested metastases of papillary thyroid carcinoma.

Papillary thyroid carcinoma is one of the common indications of thyroid surgery, $60 \%$ of PTC has lymph nodes metastases at the time of diagnosis (Baumgarten et al., 2019). The involvement of lymph nodes may increase the chance of recurrence, but in a younger patient, it does not change the prognosis. Papillary thyroid carcinoma is known to have a good prognosis and most patients can be cured if treated appropriately and early. The management of PCT is thyroidectomy followed by radioactive iodine ablation and thyroid 
replacement therapy for TSH suppression. In addition to thyroidectomy, radical neck dissection or modified radical neck dissection can be performed on a person which requires excision of tissue involved by cancer, removing all lymph nodes in the neck, as in radical neck dissection, while preserving the spinal accessory nerve in modified radical neck dissection (Aboelnaga \& Ahmed, 2015). In this case, the patient had modified bilateral neck dissection and total thyroidectomy because there were multiple lymph nodes enlargements bilateral in levels $1,2,3,4$, and 6 . The patient also has thyroid therapy replacement, with the plan to have radioiodine ablation.

The complication of thyroid surgery is hypocalcemia secondary to hypoparathyroidism, injury to the recurrent laryngeal nerve, injury to the superior laryngeal nerve neck hematoma, infection, thyrotoxic storm. Hypocalcemia is the common complication of central neck surgery especially thyroid surgery (Mejia et al., 2018; Boumgarten et al., 2019; Limaiem et al., 2019). It is due to the parathyroid glands trauma caused by direct injury during surgery, devascularization caused by obstruction of venous drainage, or incidental parathyroidectomy (Dedivitis et al., 2017). This parathyroid glands trauma will cause HPT associated with low or no production of parathyroid hormone, characterized by hypocalcemia and hyperphosphatemia. Hypocalcemia can be symptomatic and asymptomatic. The symptoms of hypocalcemia appear like the effect of the low level of serum calcium on the organ and the acuity of the onset. In acute onset the neurological symptoms will be described as paresthesia in the perioral region, hands and feet progressing to carpopedal spasm are followed by an involuntary muscle contraction accompanied by pain, hyperreflexia, and muscle spasm. The chvostek's sign on the face, present in $94 \%$ of cases of true acute hypocalcemia, and trousseau's sign are the classical sign of neurological examination (Mejia et al., 2018). In severe cases hypocalcemia can cause death due to the change of cardiac contractility and cardiac electrical conduction, laryngospasm, bronchospasm, and seizure (Mejia et al., 2018; Stack et al., 2015). Biochemically, hypocalcemia is defined as CSC for albumin concentration level $<8 \mathrm{mg} / \mathrm{dl}$. The hypoparathyroidism after thyroid surgery is defined if the CSC value $<8 \mathrm{mg} / \mathrm{dL}$ (with or without hypocalcemia symptoms) and PTH $<15 \mathrm{pg} / \mathrm{mL}$. In this case, the patient had hypocalcemia secondary to hypoparathyroidism, with CSC $5.7 \mathrm{mg} / \mathrm{dL}$ and PTH $1.37 \mathrm{pg} / \mathrm{mL}$ The acute sign of hypocalcemia were numbness in her hands and feet, progressed into carpopedal spasm, the patient also had chovstek's sign with hypotension without abnormal electrocardiography findings (Cho et al., 2014; Ritter et al., 2015).

The risk factor for HPT post thyroid surgery is bilateral central neck surgery, surgery for thyroid malignancy, parathyroid hyperplasia surgery, central compartment lymphadenectomy due to metastases, surgeon expertise, surgical technique and duration of the surgery, deficiency or insufficiency vitamin D presurgery, prior gastric bypass surgery, female, pregnancy, and lactation (Stack et al., 2015, Limaiem et al., 2019). Hypoparathyroidism after thyroid surgery can be classified as transient HPT and permanent HPT, based on the resolution time from hypocalcemia. The hypocalcemia in transient HPT will recover in 6 to 12 months after surgery and the improvement is settled without treatment, meanwhile, the hypocalcemia in permanent HPT will last more than 12 months after neck surgery (Mejia et al, 2018; Stack et al., 2015). The prevalence of transient HPT is $27 \%$ and the permanent HPT is $1 \%$ (Dedivitis et al., 2017).

In this case, the risk factor was modified bilateral central neck surgery, surgery for thyroid malignancy, central compartment lymphadenectomy due to metastases, duration of the surgery ( 5 hours and 45 minutes), and gender (female). The dissection of the central neck nodal compartment did not take the parathyroid gland due to an improvement of PTH one month after surgery from $1.37 \mathrm{pg} / \mathrm{mL}$ to $3.84 \mathrm{pg} / \mathrm{mL}$ without replacement of PTH, but for now, we still cannot diagnose whether it is transient HPT or permanent HPT yet because the chronic management for HPT continues.

The management of hypocalcemia in HPT is divided based on the onset and the symptom of hypocalcemia. According to the PTH level and CSC level in the first 24 hours after surgery, the management is divided into 3 groups 1) low risk (CSC 8-8.5 mg/dL, PTH $>15 \mathrm{pg} / \mathrm{mL}$ ), patient can be discharged from the hospital with 600 mg elemental calcium per day orally, and weekly clinical and laboratory tests; 2) medium risk (CSC 7.5-8 $\mathrm{mg} / \mathrm{dL}$ or PTH 6-15 pg/mL), patient is recommended to take elemental calcium $2400 \mathrm{mg} /$ day and calcitriol 1 $\mu \mathrm{g} /$ day in divided doses; 3 ) high risk (CSC $<7.5 \mathrm{mg} / \mathrm{dL}$ and PTH $<5 \mathrm{pg} / \mathrm{mL}$ ), patient must be given intravenous calcium gluconate $10 \%$, until it reaches the values $>7.5 \mathrm{mg} / \mathrm{dL}$, elemental calcium 3,000-6,000 mg/day and calcitriol 1.5-2 $\mu \mathrm{g} /$ day (Mejia et al., 2018; Di Maio et al., 2018). Chronic management of HPT usually is applied in the outpatient clinic. Patients who fail to show normal levels of CSC with mild symptoms or asymptomatic of hypocalcemia can get oral calcium, vitamin D supplementation, and thiazide diuretic if the blood pressure is

Arimbawa,. I. M., Paramita, A. D. P., Nuaba, I. G. A., \& Saputra, H. (2021). Management of hypocalcemia after modified bilateral radical neck dissection followed with total thyroidectomy: a case report. International Journal of Health Sciences, 5(1), 9-19. https://doi.org/10.29332/ijhs.v5n1.654 
normal or elevated, to maintain the asymptomatic hypocalcemia and avoid the complication of overtreatment which may lead to nephrolithiasis, nephrocalcinosis and renal insufficiency (Mejia et al., 2018; Stack et al., 2015; Di Maio et al., 2018).

In this case, the patient was matched with high-risk criteria of hypoparathyroidism and was given acute management for HPT. The calcium gluconate $10 \%$ was given as in adult dose, $100 \mathrm{mg} / \mathrm{kg} / \mathrm{day}$ every 8 hours followed by continuous intravenous infusion of $1 \mathrm{mg} / \mathrm{kg} /$ hour until reached value $>7.5 \mathrm{mg} / \mathrm{dL}$ on the third day. The patient was administered with calcium carbonate $3,000 \mathrm{mg} /$ day and calcitriol $0.04 \mu \mathrm{g} / \mathrm{kg} /$ day on the third day. The patient had 8 days length of stay due to repeated hypocalcemia. After discharged from the hospital patient continued the treatment by taking oral calcium, vitamin D3, and hydrochlorothiazide. The CSC showed a lower level of normal range after 6 months of therapy with elemental calcium 3,000 mg/day in 3 divided doses, vitamin D3 4,000 IU/day in 2 divided doses, and hydrochlorothiazide $25 \mathrm{mg}$ daily.

The presence of postoperative hypocalcemia especially symptomatic hypocalcemia after thyroidectomy may prolong hospitalization and the treatment of HPT remains a challenge. The mean length of stay in the patient without complication thyroidectomy in a high-volume hospital was 2 days (Limaiem et al., 2019). The best acute management of patient care relies on the early identification of patients with high or low risk for developing hypocalcemia. This information would allow timely supplementation of calcium and 1.25 hydroxyvitamin D in high-risk patients and to reduce the unnecessary serum calcium levels examinations in those with low risk (Maxwell et al., 2017; Tolone et al., 2013; Alsubhi et al., 2018). In this case, the length of stay of our patient was 8 days from the surgery due to symptomatic hypocalcemia. The calcium and PTH were checked when the symptoms occurred. The patient got routine $500 \mathrm{mg}$ calcium elemental in calcium carbonate daily. The patient also had a serial invasive procedure for biochemical evaluation. A study stated that PTH is a useful diagnostic prediction to consider the best time to initiate preventive treatment in a patient with high-risk hypocalcemia and it will reduce the duration of hospitalization and reduce the invasive procedure in those who remained normocalcemia. Based on the analysis, the results show that PTH $\leq 14$ $\mathrm{pg} / \mathrm{mL}$ by ICMA or $\leq 16 \mathrm{pg} / \mathrm{mL}$ by ECLIA that was taken 5 minutes after thyroid removal is very specific and sensible to detect patients at risk of developing hypocalcemia. This information allows the identification of patients who will need intravenous calcium infusion. Optimal sensitivity and specificity for predicting hypocalcemia (Sensitivity 93 \% (CI 95\%: 68-100), Specificity $100 \%$ (CI 95\%: 80-100) was achieved with this strategy (Freire et al., 2014).

The therapy of acute HPT is usually readily accomplished with intravenous calcium and active vitamin D analogs. There are no formal treatment guidelines for the long term management of HPT. The primary goal of chronic management is to maintain serum calcium level within an asymptomatic range and to avoid significant hypo- or hypercalcemia, to reduce the risk of symptoms, kidney stones, and ectopic soft tissue calcification, it is recommended that serum calcium and phosphorus must be maintained in the low and high normal reference ranges. Calcium is typically provided as calcium carbonate or calcium citrate. Doses ranging up to 9,450 mg per day have been reported, with most patients requiring 1,500 mg elemental calcium daily. Dosage is divided into 2 or 3 split doses to maximize absorption. Calcitriol improves intestinal calcium absorption and always required. Doses range from 0.125 to $4.0 \mathrm{mcg}$ /day, with most patients requiring 0.25 mcg daily. Vitamin D2 (ergocalciferol) or vitamin D3 (cholecalciferol) are occasionally used along with the activated metabolite of vitamin D (calcitriol) and may help to provide smoother control of calcium levels. Vitamin D therapy has the unwanted effect of increasing intestinal phosphate absorption. When severe hyperphosphatemia is problematic, intestinal phosphate binders may be necessary (Tolone et al., 2013; Alsubhi et al., 2018). Thiazide diuretics can be added to the regimen when it is difficult to control calcium or hypercalciuria $(>150 \mathrm{mg} / 24 \mathrm{~h}$ ) is a problem if blood pressure is normal or elevated. Thiazide diuretics enhance distal renal tubular calcium reabsorption, thereby increases serum calcium and reduce urinary calcium excretion. Hydrochlorothiazide (12.5-50 mg daily) can be effective. Also, a systematic review stated that a significant decrease was noted in postoperative hypocalcemia patients who received routine supplementation of oral calcium or vitamin D. The incidence decreased even more with the combined administration of both supplements. Based on the analysis, the journal commends oral calcium for all patients following thyroidectomy, with the addition of vitamin D for high-risk individuals. It is suitable for our case (Alhefdhi et al., 2013). In this case, the patient required 3,000 mg elemental calcium daily divided into 3 doses and vitamin D3 4,000 IU divided into 2 doses, and hydrochlorothiazide $25 \mathrm{mg}$ per day. The CSC and phosphate serum level reached the target after 6 months of therapy. 
From this case, we conclude that we must consider measurement of PTH at 5 minutes after total thyroidectomy to identify the high risk of hypocalcemia children and the management of post thyroidectomy with elemental calcium in combination with vitamin D or its metabolites, which are the most effective strategy. This management may result in decreased incidence of hypocalcemia, hypocalcemia symptoms, and shorter length of stay.

\section{Conclusion}

A fourteenth years old girl with papillary thyroid carcinoma (PTC) and lymph node metastases was managed with modified bilateral radical neck dissection followed by total thyroidectomy. The duration of the neck surgery was 5 hours and 45 minutes. Thirty-six hours after surgery patient had numbness localized in her hands and feet, progressed into cramps with pain and positive chvostek's sign. Physical examinations showed the hemodynamic was stable, there were no signs of a seizure and respiratory distress. Laboratory post thyroidectomy revealed critical value of hypocalcaemia $5.7 \mathrm{mg} / \mathrm{dL}$ with normal albumin $3.80 \mathrm{~g} / \mathrm{dL}$, hypomagnesium $1.43 \mathrm{mg} / \mathrm{dL}$, vitamin D $11.73 \mathrm{ng} / \mathrm{mL}$ and PTH $1.37 \mathrm{pg} / \mathrm{mL}$. In addition to the growth factor of this patient according to her height for age (HFA, CDC 2000) was within 10-25 percentile and her height was still within mid parental height, the upper-lower segment was 0.98 , there was no sign of vitamin D deficiency according to the anthropometry status. Based on the sign and symptoms, the patient was matched with highrisk criteria of hypoparathyroidism and was given acute management for hypoparathyroidism due to thyroid surgery. The calcium gluconate $10 \%$ given as in adult dose, $100 \mathrm{mg} / \mathrm{kg} /$ day every 8 hours followed by continuous intravenous infusion of $1 \mathrm{mg} / \mathrm{kg} /$ hour until reached value $>7.5 \mathrm{mg} / \mathrm{dL}$ on the third day. The patient was managed with calcium carbonate $3.000 \mathrm{mg} /$ day and calcitriol $0.04 \mu \mathrm{g} / \mathrm{kg} /$ day on the third day. Her length of stay was 8 days due to repeated hypocalcemia. After discharged from the hospital patient continued the treatment by taking oral calcium, vitamin D3, and hidroclorotiazid. The CSC showed a lower value of the normal range after 6 months of therapy with calcium elemental 3,000 mg/day in 3 divided doses, vitamin D3 4,000 IU/day in 2 divided doses, and hydrochlorothiazide 25 mg daily.

\section{Acknowledgments}

We are grateful to two anonymous reviewers for their valuable comments on the earlier version of this paper.

\footnotetext{
Arimbawa,. I. M., Paramita, A. D. P., Nuaba, I. G. A., \& Saputra, H. (2021). Management of hypocalcemia after modified bilateral radical neck dissection followed with total thyroidectomy: a case report. International Journal of Health Sciences, 5(1), 9-19. https://doi.org/10.29332/ijhs.v5n1.654
} 


\section{References}

Abboud, B., Sargi, Z., Akkam, M., \& Sleilaty, F. (2002). Risk factors for postthyroidectomy hypocalcemia. Journal of the American College of Surgeons, 195(4), 456-461. https://doi.org/10.1016/S1072-7515(02)01310-8

Aboelnaga, E. M., \& Ahmed, R. A. (2015). Difference between papillary and follicular thyroid carcinoma outcomes: an experience from Egyptian institution. Cancer biology \& medicine, 12(1), 53.

Alhefdhi, A., Mazeh, H., \& Chen, H. (2013). Role of postoperative vitamin D and/or calcium routine supplementation in preventing hypocalcemia after thyroidectomy: a systematic review and metaanalysis. The Oncologist, 18(5), 533.

Alsubhi, H. M., Yousef, F. M., Almarzouki, H. Z., \& Sindi, H. A. (2018). Effect of Vitamin D and Calcium Supplementations on Reducing the Incidence of Hypocalcemia after Thyroidectomy. World Journal of Medical Sciences, 15(2), 69-75.

Baumgarten, H. D., Bauer, A. J., Isaza, A., Mostoufi-Moab, S., Kazahaya, K., \& Adzick, N. S. (2019). Surgical management of pediatric thyroid disease: Complication rates after thyroidectomy at the Children's Hospital of Philadelphia high-volume Pediatric Thyroid Center. Journal of pediatric surgery, 54(10), 19691975. https://doi.org/10.1016/j.jpedsurg.2019.02.009

Cho, N. L., Moalem, J., Chen, L., Lubitz, C. C., Moore Jr, F. D., \& Ruan, D. T. (2014). Surgeons and Patients Disagree on the Potential Consequences of Hypoparathyroidism. Endocrine Practice, 20(5), 427-446. https://doi.org/10.4158/EP13321.0R

Dedivitis, R. A., Aires, F. T., \& Cernea, C. R. (2017). Hypoparathyroidism after thyroidectomy: prevention, assessment and management. Current opinion in otolaryngology \& head and neck surgery, 25(2), 142-146.

Di Maio, S., Soliman, A. T., De Sanctis, V., \& Kattamis, C. (2018). Current treatment of hypoparathyroidism: theory versus reality waiting guidelines for children and adolescents. Acta Bio Medica: Atenei Parmensis, 89(1), 122.

Freire, A. V., Ropelato, M. G., Ballerini, M. G., Acha, O., Bergadá, I., de Papendieck, L. G., \& Chiesa, A. (2014). Predicting hypocalcemia after thyroidectomy in children. Surgery, 156(1), 130-136. https://doi.org/10.1016/j.surg.2014.02.016

Limaiem, F., Rehman, A., \& Mazzoni, T. (2019). Cancer, papillary thyroid carcinoma (PTC). StatPearls [Internet].

Maxwell, A. K., Shonka, D. C., Robinson, D. J., \& Levine, P. A. (2017). Association of preoperative calcium and calcitriol therapy with postoperative hypocalcemia after total thyroidectomy. JAMA Otolaryngology-Head \& Neck Surgery, 143(7), 679-684.

Mejia M.G., Gonzalez-Devia, D., Fierro, F., Tapiero, M., Rojas, L., Cadena, E. (2018). Hypocalcemia posthyroidectomy: prevention, diagnosis and management. J Transl Sci, 4(2), 1-7.

Reinhardt, T. A., Lippolis, J. D., McCluskey, B. J., Goff, J. P., \& Horst, R. L. (2011). Prevalence of subclinical hypocalcemia in dairy herds. The Veterinary Journal, 188(1), 122-124. https://doi.org/10.1016/j.tvjl.2010.03.025

Ritter, K., Elfenbein, D., Schneider, D. F., Chen, H., \& Sippel, R. S. (2015). Hypoparathyroidism after total thyroidectomy: incidence and resolution.journal of surgical research,197(2), 348-353. https://doi.org/10.1016/j.jss.2015.04.059

Stack, B.J., Bimston, D.N., Bodenner, D.L., Brett, E.M., Dralle, H., Orloff, L.A., et al. (2015). American association of clinical endocrinologists and American college of endocrinology disease state clinical review: postoperative hypoparathyroidism - definition and management. Endocr Pract, 21(6), 674-83.

Tolone, S., Roberto, R., del Genio, G., Brusciano, L., Parmeggiani, D., Amoroso, V., ... \& Docimo, L. (2013). The impact of age and oral calcium and vitamin D supplements on postoperative hypocalcemia after total thyroidectomy. A prospective study. BMC surgery, 13(2), 1-6. 


\section{Biography of Authors}

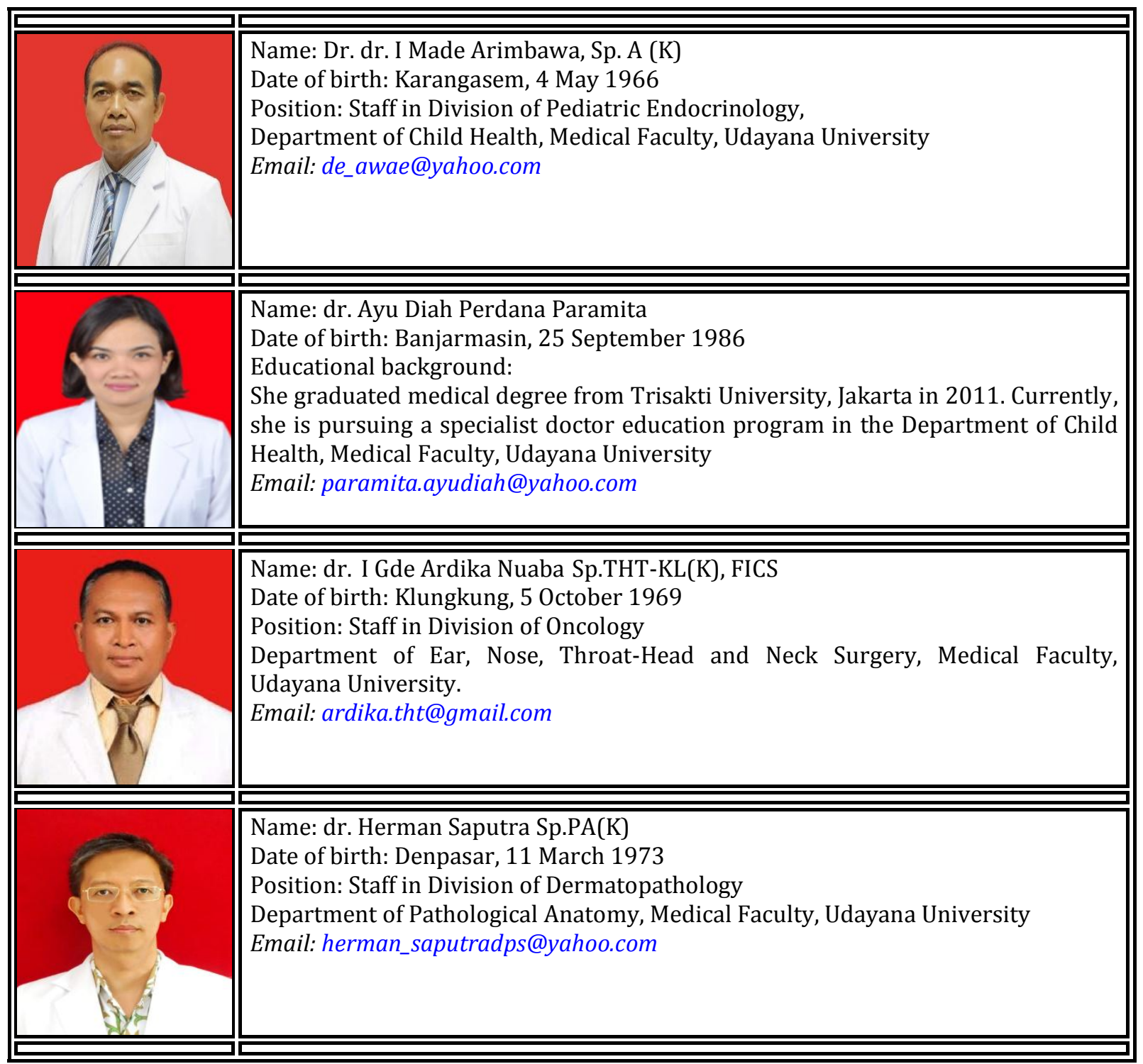

Arimbawa,. I. M., Paramita, A. D. P., Nuaba, I. G. A., \& Saputra, H. (2021). Management of hypocalcemia after modified bilateral radical neck dissection followed with total thyroidectomy: a case report. International Journal of Health Sciences, 5(1), 9-19. https://doi.org/10.29332/ijhs.v5n1.654 